\title{
Finding least fuel emission paths in a network with time-varying
}

\author{
speeds \\ Jiani Qian \& Richard Eglese* \\ Department of Management Science \\ Lancaster University Management School \\ Lancaster, LA1 4YX, U.K.
}

\begin{abstract}
This paper considers the problem of finding a route and schedule for a vehicle starting from a depot, visiting a set of customers and returning to the depot, in a time-dependent network where the objective is to minimize the greenhouse gas emissions. In this formulation, the speeds of the vehicle as well as the routes chosen are decision variables subject to limits determined by the level of congestion on the roads at the time. Two methods are proposed to find the optimal strategy for a single route. One is a time-increment based Dynamic Programming method, and the other is a new heuristic approach. In addition, a case study is carried out, which compares the performance of these methods, as well as the least polluting routes with the shortest time routes between two customer nodes.
\end{abstract}

Key words: Time-varying speeds, fuel emissions optimization, dynamic programming, heuristics

*Corresponding Author: email R.Eglese@lancaster.ac.uk

Accepted for publication in Networks 


\section{Introduction}

Current vehicle routing and scheduling software is designed to produce schedules for freight vehicles to minimize the relevant economic costs to the logistics service provider. Typically these are based on numbers of vehicles and drivers required and the distance travelled. Although the software may allow different speeds for vehicles on different types of roads, this is a rough approximation that does not take into account the different speeds on different roads at different times of day. This results in schedules where freight vehicles spend time in congested traffic, contributing to the congestion and associated environmental costs. Congestion in this paper is defined as a condition on a road network that occurs as use increases and results in lower speeds and longer journey times for the vehicles using the road network.

Although some approaches claim to produce routes that minimize pollution, they often use constant speed models that do not properly account for the effects of changing road speeds at different times of day and variations of driving speed in traffic. Congestion due to different traffic volumes and features such as long-term road works can be predicted from past experience. Information is now available from many different providers to show how long it will take to travel along different roads at different times of day and different days of the week. The data can enable the development of new vehicle routing and scheduling systems to plan schedules for distribution operations that minimize environmental damage.

This paper will focus on solving the fuel emissions optimization problem for visiting a sequence of customers in a road network with time-varying speeds. The network is made up of many short road sections, and the vehicle may travel with different speeds along different road sections, subject to maximum limits determined by the traffic congestion at different times. To our knowledge, this particular problem has not been studied in the literature. In a static network, the optimal cost for traveling along a single route, visiting several customers along the way, is the summation of the optimal travel costs between customers, which can be predetermined. However, the calculation in a time-varying network is more complicated. To obtain a least fuel emissions solution overall, it may be worthwhile having inferior solutions between some of the customers 
in order to obtain more benefit later in the journey. This is a key complication in solving the problem.

The structure of the paper is as follows. Section 2 contains a brief review of green logistics and time-varying VRPs. Then in Section 3, the method to estimate emissions from freight vehicles in terms of the Carbon Dioxide Equivalent $(\mathrm{CO} 2 \mathrm{e})$ is examined, which will be used in later calculations. Details of the problem along with some definitions will be described in Section 4. A time-increment based Dynamic Programming method and a new heuristic approach are introduced in Section 5 and Section 6. The results of a computational experiment in Section 7 compare the performances of these two methods, and provide an indication of the benefits from minimizing the fuel emissions.

\section{Literature Review}

Most of the research in VRPs is based on the assumption that attributes of the road network are constant; however, this may not be the case in the real world. The traffic volumes vary during the day especially in urban areas, hence the vehicle routing and scheduling obtained using a static model may be unreliable. This first part of this section will examine the VRP literature dealing with time-varying road networks and the references discussed are summarized in Table 1.

\begin{tabular}{|l|l|}
\hline Ziliaskopoulos and Mahmassani [1] & Van Woensel et al. [9] \\
\hline Chabini [2] & Fleischmann et al. [10] \\
\hline Dolinskaya [3] & Maden et al. [11] \\
\hline Ahn and Shin [4] & Kok et al. [12] \\
\hline Sung et al. [6] & Kim et al. [13] \\
\hline Ichoua et al. [7] & Kim et al. [14] \\
\hline Eglese et al. [8] & Haghani and Jung [15] \\
\hline
\end{tabular}

Table 1 - References for time-varying road networks

The Dynamic programming (DP) method is widely used to find the optimal routes between customer nodes. Ziliaskopoulos and Mahmassani [1] applied a DP method to calculate the shortest paths from all nodes to a given destination node for each time 
step over a time horizon in a time-varying network. The time-varying information is represented by the different travel times to pass the same arc in different periods of the day. This method is able to deal with networks where the cost is not necessarily travel time. Chabini [2] investigated the efficiency for the algorithm to compute the optimal paths between nodes in discrete dynamic networks, where the travel time is time-varying for a finite time interval, and static beyond the time horizon. A Decreasing Order of Time algorithm was proposed, where a DP recurrence is employed, to solve an "all-to-one all departure time intervals fastest/minimum cost path" problem. In this paper, it is proved that the shortest time path in a time-dependent network can be solved by using Dijkstra's algorithm with the same computational efficiency as in a static network, when unlimited waiting at nodes is allowed. Dolinskaya [3] used a DP model to find the shortest-time path for vessels, where travel times between nodes depend on the direction, location and time. In this model, it is not allowed to wait at nodes until the optimal departure time, but a similar effect can be achieved by slowing the speeds down enough so as to arrive at nodes at the optimal departure time.

Travel time has to be modeled very carefully in order to find the shortest-time route in time-varying networks. Ahn and Shin [4] modified three basic vehicle routing heuristics to allow the travel time to maintain the "first-in-first-out"(FIFO) property, and derived efficient time feasibility check routines. The FIFO property states that a vehicle departing from node $i$ at any time $t$ will not arrive at node $j$ at a later time than an identical vehicle starting from node $i$ at time $t^{\prime}$ using the same arc $a_{i j}$, where $t \leq t^{\prime}$. Providing the FIFO property holds, Dijkstra's algorithm can be adapted to find the shortest-time path between nodes efficiently [5]. Sung et al. [6] suggested a model for time-dependent networks, where the travel time was modified to satisfy the FIFO property. Therefore, an adapted Dijkstra's label-setting algorithm was able to solve the shortest paths problem. Ichoua et al. [7] proposed a model to solve a vehicle dispatching problem based on time-dependent travel speeds, with the travel time function following the FIFO property. A modified parallel tabu search algorithm is applied to solve the vehicle routing problem by minimizing the total travel times. Eglese et al. [8] model the travel time by using a similar method to that of Ichoua et al. [7], and apply Dijkstra's algorithm to construct a Road Timetable ${ }^{\mathrm{TM}}$, which includes the minimum time and the shortest-time path between locations at different starting 
times. Van Woensel et al. [9] modeled VRPs with time-dependent travel times by using a queuing approach, which is needed when traffic volumes are available and not vehicle speeds. Moreover, it is able to deal with a large number of time slots, which improves the solution quality.

Fleischmann et al. [10] presented a way of deriving time-varying travel time data from traffic information systems. Then a framework for the implementation of time-varying travel times in various vehicle routing algorithms is described. Results of computational tests indicated that the time-dependent data provide an excellent estimation of travel time, and improve the shortcomings of constant average travel times such as travel time underestimation and time window violation. A case study has been carried out by Maden et al. [11], and the results show that about 7\% of $\mathrm{CO} 2$ can be saved by considering the time-varying speeds when scheduling the vehicles and planning the routes.

When scheduling and routing vehicles in a time-varying road network, the starting time is an important factor. Experimental results of Van Woensel et al. [9] indicated that the starting time would influence the solution quality. Kok et al. [12] has proposed an ILP formulation to minimize the total duty time of a complete route, which performed as a post-processor for a Vehicle Routing Problem with Time Windows. Travel time between two customer nodes is considered as deterministic but time-dependent, and the departure time at each customer in the complete route is the decision variable. Kim et al. [13] proposed decision-making procedures to determine the minimum cost policy which includes the driver attendance time, departure times and routes in a time-varying network based on a Markov decision process formulation. The results of a case study show that savings in costs and vehicle usage for just-in-time delivery were achieved compared to the static model. Furthermore, the historical traffic information made more contributions to the savings than the real-time traffic information. Kim et al. [14] also developed a two-phase process to reduce the state space by, so enabling the computational running time to be improved. Haghani and Jung [15] applied a genetic algorithm to solve VRPs with dynamic demands in time-dependent networks. It is concluded that the performance of the dynamic routing strategy became better with the increase in the uncertainty in the traffic data.

The second part of this section considers literature concerned with climate change and 
green vehicle routing. The articles discussed are summarized in Table 2.

\begin{tabular}{|l|l|}
\hline European Environment Agency Report [16] & Bektas and Laporte [21] \\
\hline Climate Change Committee Report [17] & Ubeda [22] \\
\hline Dekker et al. [18] & Erdoğan and Miller-Hooks [23] \\
\hline Paterson [19] & Figliozzi [24] \\
\hline Hensher [20] & Figliozzi [25] \\
\hline
\end{tabular}

Table 2 - References for green vehicle routing

The Climate change due to emissions of greenhouse gases caused by human activity has been recognized as a threat to the global environment, and some actions have been taken worldwide to deal with the challenge. In 2007, the European Union (EU) declared its intention to cut greenhouse gas emissions by 20\% from the 1990 level by 2020 [16]. The $4^{\text {th }}$ carbon budget recommended by the UK Climate Change Committee [17] is to limit emissions of GHGs to 1950 MtCO2e over 2023-2027, amounting to an emission cut of $50 \%$ compared with 1990. This ambitious proposal was accepted by the UK Government in May 2011.

However, it will not be possible to achieve the ambitious goals of GHG reduction without significant progress in the transport sector, where energy use and greenhouse gas emissions are still growing [16]. Decisions in the area of logistics need to take into account environmental considerations as well as economic costs, as demonstrated by the social responsibility policies of many companies. Dekker et al. [18] has reviewed the different aspects and issues in a supply chain, where OR models can be applied to improve the environmental costs. As one of the significant parts in a supply chain, there has been increasing interest and research in vehicle routing and scheduling with respect to the environment in recent years.

Paterson [19] has developed a carbon footprint estimator, which enables carbon footprinting within the freight sector to be measured and examined. Furthermore, several fuel saving measures are recommended, and their effectiveness in improving CO2 emissions are evaluated. Hensher [20] has applied Transportation and Environment Strategy Impact Simulator (TRESIS) to evaluate the ability of instruments to reduce $\mathrm{CO} 2$ emissions. It is concluded that a mix of technology (i.e. 
fuel efficiency improvements) and pricing through a carbon tax or a variable user charge is the way forward assuming continuing use of fossil fuels.

Bektas and Laporte [21] have proposed a Pollution-Routing Problem (PRP) which takes the weight of the loads, greenhouse gas emissions, fuel, travel times, their costs as well as travel distance into account. The trade-off among the total distance, driver costs and fuel costs was studied, and the results reveal that PRP offers the potential to save costs. Ubeda [22] describes a case study concerning the environmental costs in a vehicle routing problem with backhauls. By introducing backhauls, empty-running which wastes fuel, has been avoided. The fuel emissions are evaluated based on the distance, but load factors are also considered. Erdoğan and Miller-Hooks [23] have investigated a problem that they have called a green vehicle routing problem, which involves a fleet of electric vehicles with limited battery life. Not only total distance but also recharging arrangements have to be considered when vehicle routes are scheduled.

All of the 'Green' VRPs mentioned so far have been restricted in static networks, and most of the time-varying VRP research reviewed above has focused on minimizing time related cost. However, Figliozzi [24] presents an emission minimizing VRP in a time-varying road network. The $\mathrm{CO} 2$ emissions are calculated based on a speed-dependent function. The total working time is partitioned into intervals and for each arc of the network, a set of speeds are assigned corresponding to each interval. Vehicles are assumed to travel at the specified constant speeds depending on the time of departure from the starting node of the arc and the time intervals through which the vehicle passes before reaching the end of the arc. Thus the emissions depend on the arcs selected for the routes and the departure times. Computational tests are carried out under three settings of congestion: uncongested, somewhat congested and congested. Figliozzi [24] shows that his approach can produce significant reductions in emission levels with relatively small increases in distance traveled or fleet size. A case study is described in Figliozzi [25] where the model is used for a distribution application in Portland, Oregon. The study examines how the total emissions can be influenced by congestion, speed limits set for the roads and depot location. The model assumed that a vehicle will travel at the limit of the uncongested speed. In particular, uncongested traffic speeds generally lead to lower emissions, but if free flow travel 
speeds are higher than the optimal emissions travel speed on some roads, then uncongested travel speeds can lead to increased emissions.

The goal of this paper is to explore the fuel emissions optimization problem for visiting a sequence of customers in a road network with time-varying speeds, where speeds as well as the routes are considered as decision variables.

\section{Carbon Dioxide Equivalent Estimation}

The research addressed in this paper is to control the fuel emissions by taking vehicle speeds and routes into account rather than improving the engine technologies. Carbon Dioxide Equivalent (CO2e), which evaluates various GHGs in terms of the functionally equivalent concentration of $\mathrm{CO} 2$, is used to provide an estimation of the pollution caused by Heavy Goods Vehicles (HGVs). In order to calculate CO2e, the speed-emission coefficients should be applied first to estimate the fuel consumption. A database of vehicle speed-emission factors for fuel consumption has been reported by the Department for Transport in 'Road Vehicle Emission Factors 2009' [26]. The general format of the vehicle fuel consumption function is presented as:

$$
F C(v)=k\left(a+b v+c v^{2}+d v^{3}+e v^{4}+f v^{5}+g v^{6}\right) / v
$$

where $\mathrm{v}$ is the speed in $\mathrm{km} / \mathrm{h}$ and $\mathrm{FC}(\mathrm{v})$ is the fuel consumption in litres $/ 100 \mathrm{~km}$.

Such functions are based on the average emission factor for a certain pollutant and a given type of vehicle. To calculate fuel consumption for different types of vehicles with different standards, there are different coefficients. These coefficients are measured over a range of driving cycles. Taking Euro V diesel rigid HGVs with gross weight greater than 32 tonnes as an example, the fuel consumption for every 100 kilometres travelled is given by:

$\mathrm{FC}(\mathrm{v})=0.037\left(12690+16.56 \mathrm{v}+86.87 \mathrm{v}^{2}-3.55 \mathrm{v}^{3}+0.06 \mathrm{v}^{4}-4.77 \mathrm{E}-04 \mathrm{v}^{5}+1.38 \mathrm{E}-06 \mathrm{v}^{6}\right) / \mathrm{v}$

where $\mathrm{v}$ is the speed in $\mathrm{km} / \mathrm{h}$ and $\mathrm{FC}(\mathrm{v})$ is the fuel consumption in litres $/ 100 \mathrm{~km}$. This formula is only valid when speed is between $6 \mathrm{~km} / \mathrm{h}$ and $90 \mathrm{~km} / \mathrm{h}$, as the form of the relationship is different at very low or very high speeds. 
GHG conversion factors have been proposed by the Department for Environment Food and Rural Affairs (DEFRA) [27]. The conversion factor used in this paper is to calculate $\mathrm{CO} 2 \mathrm{e}$ from the consumption of fuel, and it mainly considers the impact of $\mathrm{CO} 2, \mathrm{CH} 4$ and $\mathrm{N} 2 \mathrm{O}$. The conversion factor for diesel is $3.1787 \mathrm{~kg} \mathrm{CO} 2 \mathrm{e}$ per unit (litres) consumed, which considers both direct and indirect emissions from the combustion of the diesel by the HGVs. Indirect emissions are associated with the extraction and transport of primary fuels as well as the refining, distribution, storage and retail of finished fuels. As a result, the formula converting diesel consumption to $\mathrm{CO} 2 \mathrm{e}$ is

$$
\mathrm{CE}(\mathrm{v})=3.1787^{*} \mathrm{FC}(\mathrm{v})
$$

The calculation of (2) and (3) only takes the direct emissions of the HGVs into account. On a congested road, it can be argued that the presence of the HGV increases congestion and so has an adverse effect on the $\mathrm{CO} 2 \mathrm{e}$ emissions from other vehicles on that road, but we have not attempted to estimate these additional indirect emissions. In many places, $\mathrm{HGV}$ s form a relatively small proportion of the road traffic compared to private cars. Consequently, it is assumed that the routes and schedule chosen by HGVs will not affect the attributes of the road networks in this model, and any changes in the emissions from other traffic in the network are not included.

\section{Problem Description}

The problem can be presented on a directed graph $G=(V, A)$, where $V=\left\{1,2, \ldots, n_{\max }\right\}$ is the vertex set and $A=\left\{a_{i j}: i, j \in V, i \neq j\right\}$ is the arc set. The length of arc $a_{i j}$ is $d\left(a_{i j}\right)$. Let $d\left(a_{i j}\right)=\infty$, if there is no arc from node $i$ to node $j$. Suppose the time horizon is divided into $\mathrm{m}$ time slots $\mathrm{T}_{1}, \mathrm{~T}_{2}, \ldots, \mathrm{T}_{\mathrm{m}}$, and the upper bound of time slot $\mathrm{T}_{\mathrm{i}}$ is denoted as $\mathrm{ub}\left(\mathrm{T}_{\mathrm{i}}\right)$. The maximum speed allowed on $\operatorname{arc} \mathrm{a}_{\mathrm{ij}}$ in time slot $\mathrm{T}_{\mathrm{k}}$ is denoted $\mathrm{v}\left(\mathrm{a}_{\mathrm{ij}}, \mathrm{T}_{\mathrm{k}}\right)$.

Let $\mathrm{C}_{\text {sub }}=\left\{\mathrm{c}_{0}, \mathrm{c}_{1}, \ldots, \mathrm{c}_{\mathrm{p}}, \mathrm{c}_{\mathrm{p}+1}\right\}$ be a sequence of nodes, where $\mathrm{C}_{\text {sub }} \subseteq \mathrm{V}$. The $\mathrm{c}_{1}, \mathrm{c}_{2} \ldots, \mathrm{c}_{\mathrm{p}}$ are customer nodes, and $\mathrm{c}_{0}$ is the depot to start from. To distinguish the original node and destination, $\mathrm{c}_{\mathrm{p}+1}$ instead of $\mathrm{c}_{0}$ is used as the finishing node of the complete route, and all of the attributes of $c_{p+1}$ are the same as $c_{0}$. Each customer $\mathrm{c}_{\mathrm{i}} \in \mathrm{C}_{\text {sub }}$ is associated with a time window $\left[\mathrm{e}\left(\mathrm{c}_{\mathrm{i}}\right), \mathrm{l}\left(\mathrm{c}_{\mathrm{i}}\right)\right]$, where $\mathrm{e}\left(\mathrm{c}_{\mathrm{i}}\right)$ and $\mathrm{l}\left(\mathrm{c}_{\mathrm{i}}\right)$ are 
the lower and upper bound of the time window respectively (with $e\left(c_{0}\right)$ the earliest start time and $\mathrm{l}\left(\mathrm{c}_{\mathrm{p}+1}\right)$ the latest finish time of the journey). The service time for customer $c_{i}$ is $b\left(c_{i}\right)$. It is assumed that the total demand of the customers in set $C_{\text {sub }}$ will not exceed the vehicle capacity; moreover, a feasible solution always exists for the sequence of customers in set $\mathrm{C}_{\text {sub }}$ with respect to the time windows.

The goal is to find the least polluting strategy for a vehicle departing from the depot $c_{0}$, and going to customers $c_{1}, c_{2}, \ldots c_{p}$ in order, then going back to $c_{p+1}$. The strategy must specify the path for the vehicle as well as the speed for the vehicle on each arc of the path and any waiting time which is only allowed at customer nodes. The speed to travel along arc $\mathrm{a}_{\mathrm{ij}}$ which is denoted $\mathrm{s}\left(\mathrm{a}_{\mathrm{ij}}\right)$ is allowed to be lower than the associated speed limit, and this is another attribute of the problem presented in the paper that distinguishes it from others.

The optimization problem can be summarized formally as follows:

Determine a sequence of $\operatorname{arcs}, \mathrm{z}^{\mathrm{k}}$, and associated speeds $\mathrm{s}\left(\mathrm{z}^{\mathrm{k}}\right), \mathrm{k}=1 \ldots \mathrm{N}$, where $\mathrm{z}^{\mathrm{k}} \in \mathrm{A}$, and waiting times at customers $w\left(c_{r}\right), r=1 \ldots p$. The objective is to minimize $F$, the total $\mathrm{CO} 2 \mathrm{e}$

$$
\operatorname{Min} F=\sum_{k=1}^{N} C E\left(s\left(z^{k}\right)\right) \cdot d\left(z^{k}\right)
$$

such that the sequence of $\operatorname{arcs}, \mathrm{z}^{\mathrm{k}}, \mathrm{k}=1 \ldots \mathrm{N}$ form a path from the depot $\mathrm{c}_{0}$, going to customers $c_{1}, c_{2}, \ldots c_{p}$ in order, then going back to $c_{p+1}$. In addition, for any arc, $\mathrm{z}^{\mathrm{k}}$, on the subpath linking customers $r-1$ to $r(r=1 \ldots p+1)$ then the time taken to travel from $\mathrm{c}_{0}$ to the end of $\mathrm{z}^{\mathrm{k}^{\prime}}$ is $\mathrm{t}_{\mathrm{k}}$, and is given by

$$
t_{k^{\prime}}=\sum_{k=1}^{k^{\prime}}\left\{d\left(z^{k}\right) / s\left(z^{k}\right)\right\}+\sum_{r=1}^{m-1}\left\{b\left(c_{r}\right)+w\left(c_{r}\right)\right\}
$$

If $\mathrm{t}_{\mathrm{k}^{\prime}} \in \mathrm{T}_{\mathrm{q}}(\mathrm{q}=1 \ldots \mathrm{m})$, then $\mathrm{s}\left(\mathrm{z}^{\mathrm{k}^{\prime}}\right) \leq \mathrm{v}\left(\mathrm{a}^{\mathrm{k}^{\prime}}, \mathrm{T}_{\mathrm{q}}\right)$. 
When $\mathrm{z}^{\mathrm{k}^{\prime}}$ is the arc on the subpath that has an end node at customer $\mathrm{r}(\mathrm{r}=1 \ldots \mathrm{p}+1)$, then $\mathrm{t}_{\mathrm{k}}$, corresponds to the arrival time at customer $\mathrm{r}$ and the time window constraints imply that $\mathrm{e}\left(\mathrm{c}_{\mathrm{r}}\right) \leq \mathrm{t}_{\mathrm{k}}, \leq 1\left(\mathrm{c}_{\mathrm{r}}\right)$.

The waiting time limit can be expressed as $\mathrm{w}\left(\mathrm{c}_{\mathrm{r}}\right) \leq \mathrm{W}(\mathrm{r}=1 \ldots \mathrm{p}+1)$

\section{Time-Increment Based Dynamic Programming}

In this section, a dynamic programming (DP) method is used to solve the fuel emission optimization, where the time horizon is discretized into small segments. Hashimoto et al. [28] have used a DP to determine the optimal start times of services at customers with a fixed order in a complete route. Travel time cost functions have been introduced to relax the time window and travel time constraints. The idea of the time-increment based DP is quite similar to the approach in [28]. However, rather than a piecewise linear cost function, the cost in terms of fuel emissions in a time-varying network is more complex.

\subsection{Assumptions}

The assumptions on which this model is based are listed as follows.

- Time is discrete, and every time increment is of length $\varepsilon$.

- Waiting is only allowed at customer nodes, but the maximum waiting time at each customer cannot exceed W.

- The time spent travelling along any arc is within a range $[\varepsilon, \mu \varepsilon]$, where $\mu$ is an integer.

- The speed limit will not change until a vehicle arrives at the next arc. In other words, traffic conditions will not change quickly enough to affect the speed of the vehicle along an arc once it has started. The traffic data used in the experiment is made up of relatively short arcs which do not take a long time to traverse so this is a reasonable assumption for our data. However this means that the FIFO property will not always hold for this model.

- Each vehicle travels at a constant speed along each arc. 


\subsection{DP Recurrence}

The optimization problem for a given sequence of customers is solved in two stages, and one DP recurrence is involved in each stage. Firstly, the optimal fuel emissions for $c_{i-1}$ to $c_{i}$, where $c_{i-1}, c_{i} \in C_{\text {sub }}$, with different starting times and finishing times are computed. Secondly, the optimal fuel emissions for the complete route through all the customers are calculated.

\subsubsection{DP Recurrence for Stage One}

Define $\mathbb{C}\left(\mathrm{c}_{\mathrm{i}}, \mathrm{t}_{\text {start }}, \mathrm{t}_{\text {finish }}\right)$ as the optimal fuel emissions of travelling from customer $\mathrm{c}_{\mathrm{i}-1}$ at time $\mathrm{t}_{\text {start }}$ and arriving at customer $\mathrm{c}_{\mathrm{i}}$ at $\mathrm{t}_{\text {finish }}$. For each adjacent customer pair $\mathrm{c}_{\mathrm{i}-1}, \mathrm{c}_{\mathrm{i}} \in \mathrm{C}_{\text {sub }}$, the optimal fuel emissions with all possible starting and finishing times have to be calculated, which are used in the second step. Define ea $\left(c_{i}\right), e d\left(c_{i}\right), \operatorname{la}\left(c_{i}\right)$ and $\operatorname{ld}\left(c_{i}\right)$ as the earliest arrival time, earliest departure time, latest arrival time and latest departure time at customer node $c_{i}$ respectively, whose values can be calculated according to the time windows. Therefore, the set of possible departure times for customer $\mathrm{c}_{\mathrm{i}}$ is $\mathbb{D}_{\mathrm{i}}=\left\{\operatorname{ed}\left(\mathrm{c}_{\mathrm{i}}\right), \operatorname{ed}\left(\mathrm{c}_{\mathrm{i}}\right)+\varepsilon, \ldots, \operatorname{ld}\left(\mathrm{c}_{\mathrm{i}}\right)\right\}$, and the set of possible arrival times for customer $c_{i}$ is $\mathbb{A}_{i}=\left\{e a\left(c_{i}\right)\right.$, ea $\left.\left(c_{i}\right)+\varepsilon, \ldots, l a\left(c_{i}\right)\right\}$.

Define $f\left(i, t_{i}\right)$ as the minimum fuel emissions from the start node to node $i$ with the associated arrival time being $t_{i}$. Let $g\left(a_{i j}, t_{i}, t_{j}\right)$ be the fuel emissions along arc $a_{i j}$ when the departure time from node $i$ and arrival time at node $j$ are $t_{i}$ and $t_{j}$ respectively. According to the last assumption in 5.1, the constant speed along arc $\mathrm{a}_{\mathrm{ij}}$ can be calculated as

$$
s\left(a_{i j}\right)=d\left(a_{i j}\right) /\left(t_{j}-t_{i}\right)
$$

The maximum speed for arc $a_{i j}$ varies with time. When the starting time is in one time slot and finishing is in another, the finishing time of the arc is considered as the determining factor in this model. In other words, $s\left(a_{i j}\right) \leq v\left(a_{i j}, T_{k}\right)$, where $\mathrm{ub}\left(\mathrm{T}_{\mathrm{k}-1}\right) \leq \mathrm{t}_{\mathrm{j}}<\mathrm{ub}\left(\mathrm{T}_{\mathrm{k}}\right)$. For arcs remaining in one time slot, using starting time or finishing time makes no difference. The formula to compute $g\left(a_{i j}, t_{i}, t_{j}\right)$ is 


$$
\begin{gathered}
g\left(a_{i j}, t_{i}, t_{j}\right)=d\left(a_{i j}\right) * \operatorname{CE}\left(s\left(a_{i j}\right)\right) \text {, if } s\left(a_{i j}\right) \leq v\left(a_{i j}, T_{k}\right) ; \\
=\infty, \text { otherwise, }
\end{gathered}
$$

where $\mathrm{ub}\left(\mathrm{T}_{\mathrm{k}-1}\right) \leq \mathrm{t}_{\mathrm{j}}<\mathrm{ub}\left(\mathrm{T}_{\mathrm{k}}\right)$, and $\mathrm{CE}\left(\mathrm{s}_{\mathrm{ij}}\right)$ is the CO2e emission function defined in Section 3.

The following DP recurrence will be used to update $f\left(j, t_{j}\right)$ via iterations. The value of $f\left(j, t_{j}\right)$ can be considered as the upper bound of the minimum fuel emissions from the start node to node $\mathrm{j}$ with the arrival time being $\mathrm{t}_{\mathrm{j}}$. The iterations will stop when the value of $f\left(j, t_{j}\right)$ cannot be reduced anymore.

$$
f\left(j, t_{j}\right)=\min _{i} \min _{t_{i} \in\left\{t_{j}-\mu \varepsilon, \ldots, t_{j}-2 \varepsilon, t_{j}-\varepsilon\right\}}\left(f\left(i, t_{i}\right)+g\left(a_{i j}, t_{i}, t_{j}\right)\right) .
$$

With the original node being $\mathrm{c}_{\mathrm{i}-1}$ and the starting time being $\mathrm{t}_{\text {start }}$, the value of $\mathbb{C}\left(c_{\mathrm{i}}, \mathrm{t}_{\text {start }}, \mathrm{t}_{\text {finish }}\right)$ can be obtained as

$$
\mathbb{C}\left(c_{i}, t_{\text {start }}, t_{\text {finish }}\right)=f\left(c_{i}, t_{\text {finish }}\right)
$$

\subsubsection{DP Recurrence for Stage Two}

Define $\mathbb{F}\left(c_{i}, t_{\text {finish }}\right)$ as the optimal fuel emissions from the depot to customer $c_{i}$ with the arrival time at $c_{i}$ being $t_{\text {finish }}$. The following DP recurrence will be utilised to calculate the optimal fuel emissions for the complete route.

$$
\mathbb{F}\left(c_{i}, t_{\text {finish }}\right)=\min _{b\left(c_{i}\right) \leq t_{i-1}-t_{i-1} \leq W+b\left(c_{i}\right)}\left\{\mathbb{F}\left(c_{i-1}, t_{i-1}\right)+\mathbb{C}\left(c_{i}, t^{\prime}{ }_{i-1}, t_{\text {finish }}\right)\right\} .
$$

Waiting is allowed at customer nodes, therefore the departure time at customer i-1 denoted as $\mathrm{t}_{\mathrm{i}-1}^{\prime}$ does not necessary equal the arrival time plus the service time; nevertheless, waiting more than $\mathrm{W}$ is prohibited.

\subsection{Complexity of the Algorithm}

The efficiency of the algorithm depends on the number of time intervals, the number of customer nodes and the number of calculations with formula (7). Ziliaskopoulos and Mahmassani [1] have proved that for any specified $t_{\text {start }}$, the upper bound of the total 
iteration number for calculating $\mathbb{C}\left(\mathrm{c}_{\mathrm{i}}, \mathrm{t}_{\text {start }}, \mathrm{t}_{\text {finish }}\right)$ for all $\mathrm{c}_{\mathrm{i}}$ and $\mathrm{t}_{\text {finish }}$ is $|\mathrm{V}|^{3}\left(\frac{\mathrm{lh}}{\varepsilon}\right)^{2}$, where $|\mathrm{V}|$ is the total number of nodes in the network and $\mathrm{lh}$ is the total length of the time horizon. Nevertheless, the number of time intervals used in the iterations in stage one with a specified starting time $t_{\text {start }}$ equals $\left(l a\left(c_{i}\right)-t_{\text {start }}\right) / \varepsilon$, which is smaller than $\frac{\text { lh }}{\varepsilon} \cdot \mathbb{C}\left(c_{i}, t_{\text {start }}, t_{\text {finish }}\right)$ has to be calculated for all $t_{\text {start }} \in \mathbb{D}_{i-1}$, which has an upper bound of $\frac{\mathrm{lh}}{\varepsilon}$. Thus, the upper bound of the number of iterations performed to find optimal solutions between two customer nodes with all combinations of starting time and finishing time is $|\mathrm{V}|^{3}\left(\frac{\mathrm{lh}}{\varepsilon}\right)^{3}$. There are $\mathrm{p}$ customers plus one depot on the path, hence the upper bound of the total iterations in stage one is $|\mathrm{V}|^{3}\left(\frac{\mathrm{lh}}{\varepsilon}\right)^{3}(\mathrm{p}+1)$. However, in stage one, not only $\mathbb{C}\left(\mathrm{c}_{\mathrm{i}}, \mathrm{t}_{\text {start }}, \mathrm{t}_{\text {finish }}\right)$ is calculated, but also a $|\mathrm{V}| \times\left(\frac{\mathrm{lh}}{\varepsilon}\right) \times\left(\frac{\mathrm{lh}}{\varepsilon}\right)$ dimensional matrix of the optimal fuel emissions from customer node $c_{i-1}$ to all the other nodes with specified starting and finishing time is obtained. This information can be stored and used for other paths with the same customers but in a different sequence.

In stage two, the DP recurrence will be performed for each customer plus the depot for every time interval, so the upper bound of the number of iterations in the second stage is $\frac{\mathrm{lh}}{\varepsilon}(p+1)$. With the time window constraint, the number of feasible finishing times for each customer node may be much smaller than $\frac{\mathrm{lh}}{\varepsilon}$.

According to the analysis, the complexity of the algorithm depends largely on the number of time intervals. An increase in the length of the time increment will reduce the number of time intervals over the same time horizon. However, this would reduce the quality of the selectable speeds, especially for short arcs. Going through an arc $\mathrm{a}_{\mathrm{ij}}$ whose length is $d\left(a_{i j}\right)$, with allowed times $\varepsilon, 2 \varepsilon, \ldots, \mu \varepsilon$ respectively, the corresponding speeds would be $d\left(a_{i j}\right) / \varepsilon, d\left(a_{i j}\right) / 2 \varepsilon, \ldots, d\left(a_{i j}\right) / \mu \varepsilon$. These speeds may be low and not environmentally friendly with small $\mathrm{d}\left(\mathrm{a}_{\mathrm{ij}}\right)$ but relatively big $\varepsilon$.

For example, the possible speeds to travel along an arc of length 50 meters are $36 \mathrm{~km} / \mathrm{h}$, $18 \mathrm{~km} / \mathrm{h}, 12 \mathrm{~km} / \mathrm{h}$ or $9 \mathrm{~km} / \mathrm{h}$, when the time increment is $5 \mathrm{~s}$. The average emission-saving speed is around $65 \mathrm{~km} / \mathrm{h}$, which in this case is not available. In the real road network, there are lots of arcs whose distance is less than $50 \mathrm{~m}$, thus the number of selectable speeds for those arcs would be even fewer, and the fuel emissions would be 
overestimated. The speed $60 \mathrm{~km} / \mathrm{h}$ will become an available option when the time increment is reduced to $3 \mathrm{~s}$. However, the number of time intervals over an hour would increase from 720 to 1200 . Actually, 5 s is already a small time increment, considering a planning horizon which may be several hours. Consequently, the length of time increment should be determined very carefully in practice.

\section{New Heuristic Method}

In the time increment based DP algorithm, the optimal fuel emissions from the original node to every other node is computed, and many of them turn out to be irrelevant to the final solution, and hence lots of computational time is wasted. Consequently, a heuristic method is developed, which contains a Route Selection (RS) process and a Speed Adjustment (SA) process. For each route proposed by the RS process, the SA process will be applied to modify the speeds of the associated arcs, and the route and speeds lead to the minimum fuel emissions will be considered as the final solution.

\subsection{Assumptions}

The assumptions on which this model is based are listed as follows.

- Speed limit will not be changed within a time slot.

- Waiting is only allowed at customer nodes, but the maximum waiting time at each customer cannot exceed W.

- Vehicle travels with a constant speed along an arc until the time slot is changed, which allows the First In First Out (FIFO) property to be held. This is different from the model developed in Section 5, and is more consistent with the real traffic situation.

\subsection{Route Selection Process}

The real road network is very complicated, and there are a large number of possible routes that go through a set of customer nodes. It takes too long to evaluate the fuel emissions of all the routes, so which ones should be selected as candidates? Should the criterion be the shortest distance route, the shortest time route or some other route? An approach called the Adaptive Searching (AS) method is developed to look for promising routes to be candidates. 


\subsubsection{Basic Ideas of the AS method}

The idea of the AS method is to generate a set of possible routes which are good candidates for one that will minimize fuel emissions when different speeds are allowed on each arc in the path. This is done by finding the shortest route paths in terms of time when different arcs are assigned different artificial speed limits and it is assumed that the vehicle travels at the artificial speed limits. The process tends to generate paths that are not very long in distance or highly congested and so make suitable potential candidates.

The following example shows how different routes can be obtained by selecting the shortest time route, based on different artificial speed limits. A network shown as in Fig. 1 is made up of six arcs, and the length of each arc is 1 . Speed limits for AB and BC are $1 / 2$, for $\mathrm{AC}$ is $1 / 6$ and $\mathrm{AD}, \mathrm{DE}$ and $\mathrm{CE}$ are 1 . The shortest time route from $\mathrm{A}$ to $\mathrm{C}$ is A-D-E-C, the shortest distance route is A-C. The fuel emissions at speeds of $1 / 6,1 / 2$ and 1 are taken to be 2.5, 1 and 1 respectively per unit of distance travelled. So the least polluting route is A-B-C, which is neither the shortest time route nor the shortest distance route. One way to make A-B-C become a candidate route is to search for the shortest time route with the restriction that the maximum speed for all of the arcs should be no more than $1 / 2$. Thus, the travel time along A-D-E-C is 6 time units, while the travel time along A-B-C is 4 time units. Consequently, the fastest route becomes A-B-C. Furthermore, the shortest time route would be the shortest distance route A-C, if the artificial speed limit is made $1 / 6$ for all of the arcs.

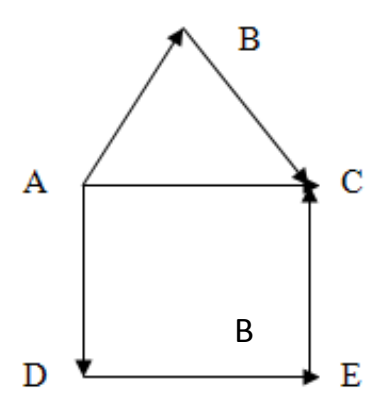

Fig.1 Constructed Network with Six Arcs 


\subsubsection{Application of the AS method}

The algorithm utilized in the route selection process is analogous to the one developed by Eglese et al. [8] to produce the Road Timetable ${ }^{\mathrm{TM}}$. Define $\Xi=\left\{\xi_{\mathrm{r}}: \mathrm{r}=1,2, \ldots, \mathrm{q}\right\}$ as a set of artificial speed restrictions, which are not necessarily ordered in magnitude. When $\xi_{\mathrm{r}} \in \Xi$ is applied, the maximum speed allowed to travel along arc $\mathrm{a}_{\mathrm{ij}}$ in time slot $\mathrm{T}_{\mathrm{k}}$ will be modified to $\xi_{\mathrm{r}}$, if $\mathrm{v}\left(\mathrm{a}_{\mathrm{ij}}, \mathrm{T}_{\mathrm{k}}\right)>\xi_{\mathrm{r}}$. With any specified $\xi_{\mathrm{r}}$, AS is applied from the starting node $c_{0}$ (depot) with the given departure time $t_{0}$ to search for the shortest-time route for each adjacent customer pair $c_{i}, c_{i+1}$, for $i=0,1, \ldots, p$. This method produces up to q potential routes to be used.

\subsection{Speed Adjustment Process}

One of the differences between time-varying VRPs for fuel emission optimization and conventional VRPs is that speed as well as the route has to be taken into account. Dynamic programming is a useful way to calculate the optimal speed travelling along a route, but the large number of iterations leads to a long running time. Therefore, an Approximate Dynamic Programming (ADP) algorithm is developed in the Speed Adjustment (SA) process to modify the speeds along the candidate route found by the route selection process.

\subsubsection{Basic Ideas of the ADP method}

The general idea of the ADP algorithm is to move arcs on a path between time slots. An arc is said to belong to a time slot if the starting time of the arc is within the time slot. Since the average speed limit for a specific arc is assumed to be the same within a time slot, it is necessary to move an arc from a time slot with low maximum speed to one with higher maximum speed in order to avoid congestion on the arc. Arcs are moved from one time slot to another via speed-control of a series of predecessors.

As illustrated in Fig. 2, the horizontal axis represents time rather than distance. Along with the change of speed, the time to travel along an arc is changed, which is reflected by the length of the arc in the horizontal axis. Since the length of time slots is fixed, increasing the length of an arc may 'push' later arcs to the next time slot. For instance, there are two strategies to pass the same path. With the first strategy, arcs 1, 2, 3, 4 are 
put into the first time slot, arcs 5, 6, 7 are put into the second time slot, and arcs 8, 9 are put into the third time slot. With the second strategy, where the speeds are lower than the first one for $\operatorname{arcs} 1,2$ and 3, the lengths of $\operatorname{arcs} 1,2,3$ are increased; as a result, arc 4 is pushed to the second time slot. Moreover, the fourth time slot is needed to include arc 9, where the speed limit for arc 9 becomes higher, and so the time to travel along arc 9 is reduced.

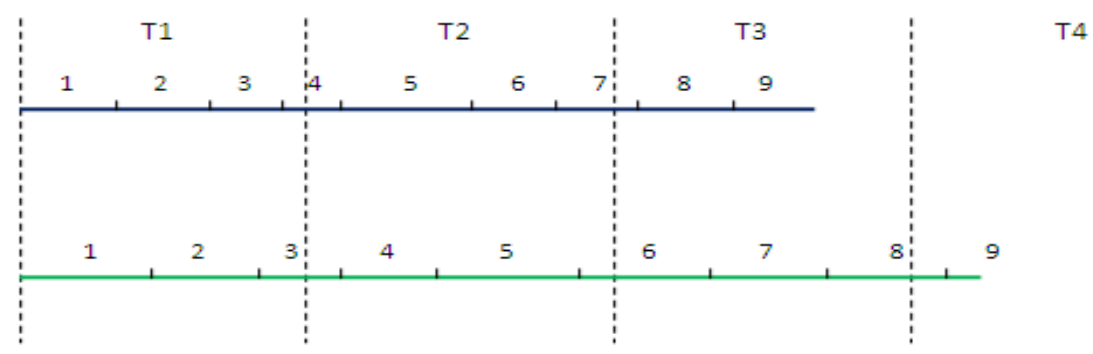

Fig. 2 The same path with different speed strategies

\subsubsection{Process of ADP method}

In the following explanation, length of an arc means the time to travel along the arc rather than the distance. Besides, the customer nodes are treated as customer arcs with length being service time plus waiting time. The ADP method starts from applying two strategies to find the minimum and maximum number of time slots required for the given path, one is travelling as fast as possible, and the other is travelling as slowly as possible, where $\zeta$ is considered as an acceptable minimum speed. Speeds of vehicles travelling on arcs across time slots are modified to maintain the FIFO property, thus the number of time slots required under the travelling as fast as possible scenario is a lower bound for the specified route, denoted as $\mathrm{lb}$, and waiting is not allowed in this case. When travelling as slowly as possible, the speed of each arc is reduced to $\zeta$, and waiting time at each customer is W. The number of the total time slots required in this case is an upper bound denoted as ub.

Let $\mathrm{TS}=\left\{\mathrm{T}^{1}, \mathrm{~T}^{2}, \ldots, \mathrm{T}^{\mathrm{lb}}, \ldots, \mathrm{T}^{\mathrm{ub}}\right\}$ be the set of time slots covered when a vehicle travels as slowly as possible along a given path $P=\left\{a^{1}, a^{2}, \ldots, a^{\varrho}\right\}$ for starting time $t_{0}$. The superscripts of the last arc in each time slot by applying the 'fast' and 'slow' strategies are recorded separately. Define idx_fast $\left(\mathrm{T}^{\mathrm{i}}\right)$ and $\operatorname{idx} \_\operatorname{slow}\left(\mathrm{T}^{\mathrm{i}}\right)$ as the index of the last arc in time slot $\mathrm{T}^{\mathrm{i}}$ by travelling as fast as possible and as slowly as 
possible respectively. Iterations start from the initial state, where travelling as fast as possible is followed. In order to improve fuel emissions, the speed will be slowed down, which will push some arcs from the current time slot to the next one. This process will be performed iteratively from the first time slot occupied, until the index of the last arc remaining in the current time slot $\mathrm{T}^{\mathrm{i}}$ equals idx_slow $\left(\mathrm{T}^{\mathrm{i}}\right)$, and then $\operatorname{arcs}$ in the next time slot will be dealt with.

\subsubsection{DP Recurrence}

Define $\mathrm{h}\left(\mathrm{i}, \mathrm{mv}\left(\mathrm{T}^{\mathrm{i}}\right)\right)$ as the optimal fuel emission from the start to the end of time slot $\mathrm{T}^{\mathrm{i}}$, when $\operatorname{mv}\left(\mathrm{T}^{\mathrm{i}}\right)$ arcs are moved out of time slot $\mathrm{T}^{\mathrm{i}}$. For $\mathrm{i}=1,2, \ldots, \mathrm{ub}, 0 \leq$ $\operatorname{mv}\left(\mathrm{T}^{\mathrm{i}}\right) \leq \mathrm{idx} \_$fast $\left(\mathrm{T}^{\mathrm{i}}\right)-\mathrm{idx} \_$slow $\left(\mathrm{T}^{\mathrm{i}}\right)$. The index of the last arc remaining in time slot $\mathrm{T}^{\mathrm{i}}$ is $\mathrm{idx} \_$fast $\left(\mathrm{T}^{\mathrm{i}}\right)-\operatorname{mv}\left(\mathrm{T}^{\mathrm{i}}\right)$, when $\operatorname{mv}\left(\mathrm{T}^{\mathrm{i}}\right)$ arcs are removed, thus in other words, $\mathrm{h}\left(\mathrm{i}, \mathrm{mv}\left(\mathrm{T}^{\mathrm{i}}\right)\right)$ is the optimal fuel emissions from the start to the end of arc with index idx_fast $\left(\mathrm{T}^{\mathrm{i}}\right)-\operatorname{mv}\left(\mathrm{T}^{\mathrm{i}}\right)$, when the starting time of this arc and the next arc are in $\mathrm{T}^{\mathrm{i}}$ and $\mathrm{T}^{\mathrm{i}+1}$ respectively.

For $\mathrm{i}=1,2, \ldots, \mathrm{ub}$, the following DP recurrence is used to calculate $\mathrm{h}\left(\mathrm{i}, \mathrm{mv}\left(\mathrm{T}^{\mathrm{i}}\right)\right)$, and $\mathrm{h}(0,0)=0$. We have that

$$
h\left(i, \operatorname{mv}\left(T^{i}\right)\right)=\min _{\operatorname{mv}\left(T^{i-1}\right)}\left(h\left(i-1, \operatorname{mv}\left(T^{i-1}\right)\right)+r\left(a^{x}, a^{y}\right)\right),
$$

where $0 \leq m v\left(\mathrm{~T}^{\mathrm{i}-1}\right) \leq \mathrm{idx} \_$fast $\left(\mathrm{T}^{\mathrm{i}-1}\right)-\mathrm{idx} \_\operatorname{slow}\left(\mathrm{T}^{\mathrm{i}-1}\right), \quad \mathrm{x}=\mathrm{idx} \_$fast $\left(\mathrm{T}^{\mathrm{i}-1}\right)-$ $\operatorname{mv}\left(\mathrm{T}^{\mathrm{i}-1}\right)+1$, and $\mathrm{y}=\mathrm{idx} \_$fast $\left(\mathrm{T}^{\mathrm{i}}\right)-\operatorname{mv}\left(\mathrm{T}^{\mathrm{i}}\right)$. The quantity $r\left(\mathrm{a}^{\mathrm{x}}, \mathrm{a}^{\mathrm{y}}\right)$ is defined as the fuel emissions from $\operatorname{arc} \mathrm{a}^{\mathrm{x}}$ to $\operatorname{arc} \mathrm{a}^{\mathrm{y}}$. It is calculated according to the speeds along arcs in time slot $\mathrm{T}^{\mathrm{i}}$, which are determined by a heuristic method explained in Section 6.3.4.

Let $f_{p}$ be the optimal fuel emissions for a complete route $p$. For $l b \leq i \leq u b$, $\operatorname{idx} \_f a s t\left(T^{i}\right)=\varrho$, which is the index of the last arc in the path; and $h(i, 0)$ is the optimal fuel emissions of the complete path with the finishing time at time slot $\mathrm{T}^{\mathrm{i}}$. Therefore, the value of $f_{p}$ can be obtained by

$$
f_{p}=\min _{l b \leq i \leq u b}(h(i, 0)) .
$$




\subsubsection{Heuristic Method for Covering Time Slack}

In the Time-Increment Based Dynamic Program algorithm, we have to compare all the different options of speeds for each arc, which is quite time consuming; therefore, a heuristic method is proposed to modify the speed so as to 'push' some arcs to the next time slot, and it will be called as a procedure in the ADP method.

Time slack is defined as the time between the starting of an arc and the upper bound of the time slot it belongs to. Let $\operatorname{slk}\left(\mathrm{a}^{\mathrm{k}}, \mathrm{i}, \mathrm{mv}\left(\mathrm{T}^{\mathrm{i}}\right)\right)$ be the time slack between $\operatorname{arc} \mathrm{a}^{\mathrm{k}}$ and the upper bound of time slot it belonged to, when $\operatorname{mv}\left(\mathrm{T}^{\mathrm{i}}\right)$ arcs are moved out of time slot $\mathrm{T}^{\mathrm{i}}$. Once $\mathrm{h}\left(\mathrm{i}, \mathrm{mv}\left(\mathrm{T}^{\mathrm{i}}\right)\right)$ is obtained, $\operatorname{slk}\left(\mathrm{a}^{\mathrm{k}}, \mathrm{i}, \operatorname{mv}\left(\mathrm{T}^{\mathrm{i}}\right)\right)$ for $\mathrm{k}>\operatorname{idx} x_{-} \operatorname{fast}\left(\mathrm{T}^{\mathrm{i}}\right)-$ $\operatorname{mv}\left(\mathrm{T}^{\mathrm{i}}\right)$ in the next time slot $\mathrm{T}^{\mathrm{i}+1}$ will be calculated. It is additionally assumed that the starting time of the arc will exactly equal the lower bound of that time slot, when an arc is pushed to a time slot. Hence, pushing the arc $\mathrm{a}^{\mathrm{y}+1}$ to time slot $\mathrm{T}^{\mathrm{i}+1}$ is equivalent to extending the length from $\operatorname{arc} \mathrm{a}^{\mathrm{x}}$ to $\operatorname{arc} \mathrm{a}^{\mathrm{y}}$ to cover the time slack $\operatorname{slk}\left(\mathrm{a}^{\mathrm{y}+1}, \mathrm{~T}^{\mathrm{i}-1}, \mathrm{mv}\left(\mathrm{T}^{\mathrm{i}-1}\right)\right)$. Define $\overline{\text { slk }}$ as the remaining time slack to cover, and initially, $\overline{\mathrm{slk}}=\operatorname{slk}\left(\mathrm{a}^{\mathrm{y}+1}, \mathrm{~T}^{\mathrm{i}-1}, \mathrm{mv}\left(\mathrm{T}^{\mathrm{i}-1}\right)\right)$.

There are two ways to cover $\overline{s l k}$. One is slowing speeds, and the other is waiting at customer nodes. The rules applied in this model are:

- $\quad$ Reduce the speeds to the optimal speed under free flow first.

- If the time slack cannot be covered, then wait at customer nodes subject to the maximum waiting time constraint.

- If the time slack cannot be covered, then further reduce the speeds.

\subsubsection{Speed Reduction}

In order to make the vehicle travel smoothly, several segments are set up according to the shape of the speed-fuel curve. Let $\Pi=\left\{\pi_{1}, \pi_{2}, \ldots, \pi_{z}\right\}$ be a set of critical speeds, where $\pi_{1}$ is the optimal speed under free flow. Since the shape of the speed-fuel consumption curve, illustrated in what follows, is flat near the optimal speed, and becomes steep along with the decrease of speed, the difference between $\pi_{i}$ and $\pi_{i-1}$ 
will be increased along with the decrease of $i$. For instance, the critical speeds can be set as $\Pi=\{65,45,35,30\}$ according to Fig. 3 .

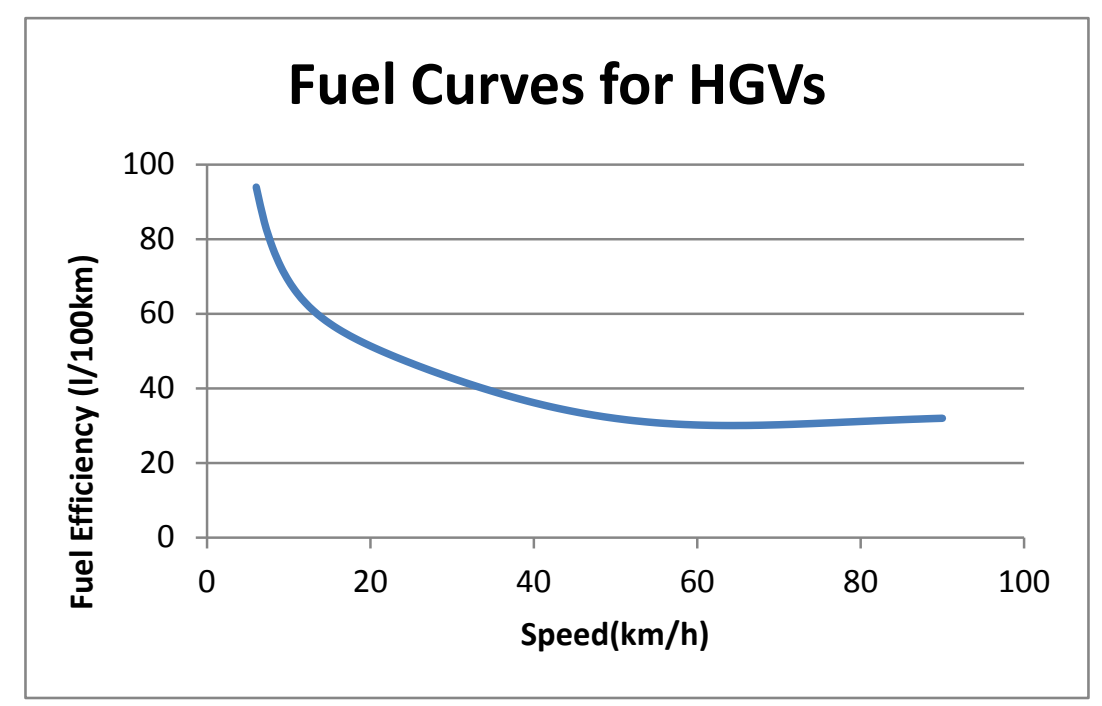

Fig. 3 Speed-Fuel Consumption curve for Euro V diesel rigids HGVs $>32$ tones

Define $s\left(a^{k}\right)$ as the speed along arc $a^{k}$. Initially, $s\left(a^{k}\right)=v\left(a^{k}, T^{i}\right)$, for $x \leq k \leq y$. Then, the value of $s\left(\mathrm{a}^{\mathrm{k}}\right)$ will be updated following a speed reduction process with critical speed $\pi_{j}$. Only these arcs with speeds exceeding $\pi_{j}$ will be considered. Define $\overline{\mathrm{s}}$ as the new speed required for arc $\mathrm{a}^{\mathrm{k}}$ to cover $\overline{\mathrm{slk}}$, and

$$
\mathrm{d}\left(\mathrm{a}^{\mathrm{k}}\right) / \overline{\mathrm{s}}-\mathrm{d}\left(\mathrm{a}^{\mathrm{k}}\right) / \mathrm{s}\left(\mathrm{a}^{\mathrm{k}}\right)=\overline{\mathrm{slk}}
$$

By solving (12),

$$
\overline{\mathrm{s}}=\frac{1}{\overline{\mathrm{slk}} / \mathrm{d}\left(\mathrm{a}^{\mathrm{k}}\right)+1 / \mathrm{s}\left(\mathrm{a}^{\mathrm{k}}\right)} .
$$

Since the critical speed is a barrier to prevent speed being reduced too quickly, speed can only be slowed down to $\pi_{j}$. If $\bar{s} \geq \pi_{j}$, then $s\left(a^{k}\right)=\bar{s}$. Thus, it is enough to cover the time slack, and the speed reduction process will stop. Otherwise, the speed of arc $\mathrm{a}^{\mathrm{k}}$ can only be reduced to $\pi_{\mathrm{j}}$, and the remaining time slack can be updated as follows:

$$
\overline{\mathrm{slk}}=\overline{\mathrm{slk}}-\left(\mathrm{d}\left(\mathrm{a}^{\mathrm{k}}\right) / \pi_{\mathrm{j}}-\mathrm{d}\left(\mathrm{a}^{\mathrm{k}}\right) / \mathrm{s}\left(\mathrm{a}^{\mathrm{k}}\right)\right)
$$

and then 


$$
\mathrm{s}\left(\mathrm{a}^{\mathrm{k}}\right)=\pi_{\mathrm{j}}
$$

Therefore, the process will be repeated for next arc with speed greater than $\pi_{j}$ until the time slack is covered. If the time slack still cannot be covered after all of the arcs in time slot $\mathrm{T}^{\mathrm{i}}$ have been checked, the process will be repeated from arc $\mathrm{a}^{\mathrm{x}}$ again with critical speed being $\pi_{j+1}$.

The following example demonstrates how the speed reduction process works for a path with 7 arcs shown in Fig. 4.

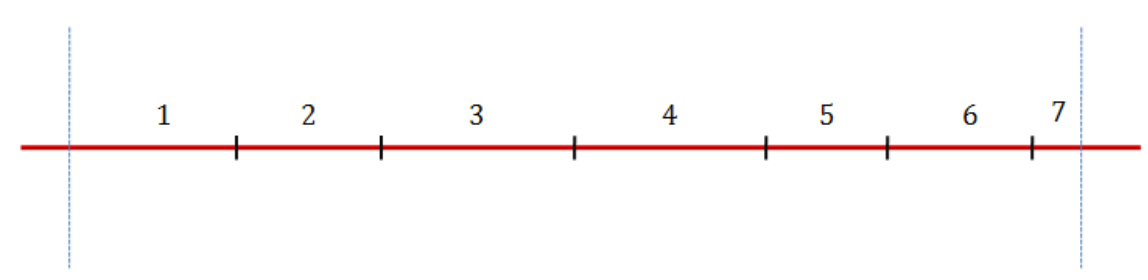

Fig. 4 Example for speed slowing down process

The speed limits for arcs 1, 2 and 3 are $54 \mathrm{~km} / \mathrm{h}, 72 \mathrm{~km} / \mathrm{h}$ and $90 \mathrm{~km} / \mathrm{h}$ respectively, and the corresponding distances are $0.6 \mathrm{~km}, 1.8 \mathrm{~km}$ and $1 \mathrm{~km}$. The time slack of arc 7 is $20 \mathrm{~s}$, which is going to be covered by slowing down the speeds of arcs 1 to 6 . The critical speeds are set as $65,45,35$ and $30 \mathrm{~km} / \mathrm{h}$. Without the critical speeds, the time slack will be covered by reducing the speed of arc 1 to $36 \mathrm{~km} / \mathrm{h}$. However, with the critical speeds, arc 2 is the first arc to be considered, because the speed of arc 1 is lower than $65 \mathrm{~km} / \mathrm{h}$. The starting time of arc 7 will be postponed for 10s by slowing the speed of arc 2 to $65 \mathrm{~km} / \mathrm{h}$, Another $10 \mathrm{~s}$ is required to be covered, so the speed of arc 3 will be reduced to $72 \mathrm{~km} / \mathrm{h}$. Thus, the time slack is covered with a smoother strategy, compared to the strategy that only reduces the speed of arc 1 to $36 \mathrm{~km} / \mathrm{h}$.

\subsubsection{Waiting}

If the time slack cannot be covered by reducing the speeds to $\pi_{1}$, waiting at a customer will be considered provided there is a customer arc in the current time slot $\mathrm{T}^{\mathrm{i}}$. Covering time slack by waiting at customer nodes is more straightforward than reducing speeds. Define $\operatorname{wt}\left(\mathrm{a}^{\mathrm{k}}\right)$ as the waiting time at customer $\operatorname{arc} \mathrm{a}^{\mathrm{k}}$. Initially, $\operatorname{wt}\left(\mathrm{a}^{\mathrm{k}}\right)=0$ for $\mathrm{x} \leq$ $\mathrm{k} \leq \mathrm{y}$. Then, for a customer $\operatorname{arc} \mathrm{a}^{\mathrm{k}}, \operatorname{wt}\left(\mathrm{a}^{\mathrm{k}}\right)=\overline{\mathrm{slk}}$, if $\overline{\mathrm{slk}} \leq \mathrm{W}$. Hence the time slack is 
covered, i.e. $\overline{\text { slk }}=0$. Otherwise, $\operatorname{wt}\left(\mathrm{a}^{\mathrm{k}}\right)=\mathrm{W}$, and $\overline{\mathrm{slk}}=\overline{\mathrm{slk}}-\operatorname{wt}\left(\mathrm{a}^{\mathrm{k}}\right)$. The time slack has not yet been covered, thus the process will be repeated for the next customer arc if there is any; or the speed reduction process will be repeated from $\operatorname{arc} \mathrm{a}^{\mathrm{x}}$ again with critical speed being $\pi_{2}$.

\section{Computational Experiments with Real Traffic Data}

\subsection{Introduction}

A study is carried out, which is based on 14 customers and a depot located around Bristol. In this section, the new heuristic approach (NHA) is compared with the time-increment based DP (TIBDP). Moreover, the least polluting routes are compared with the fastest route retrieved from the Road Timetable ${ }^{\mathrm{TM}}$. All the results are generated in the form similar to the Road Timetable ${ }^{\mathrm{TM}}$, which are the optimal strategies between a depot and a customer or between two customers, thus there are 210 routes evaluated. The time-varying speed limit data is also the same as that used by the Road Timetable $^{\mathrm{TM}}$. There are 15 time slots to cover a $24 \mathrm{~h}$ period. Midnight and $8 \mathrm{am}$ are used as the starting times to represent off-peak time and peak time.

\subsection{Result Analysis}

\subsubsection{NHA compared with TIBDP}

The main parameters for the two methods are as follows.

Time-Increment Based DP:

Time increment is $5 \mathrm{~s}$; time spent on an arc is between $5 \mathrm{~s}$ and $10 \mathrm{~min}$; and time spent on whole journey should be within $1.5 \mathrm{~h}$.

New Heuristic Approach:

Artificial speed restrictions for the routes selection process $(\mathrm{km} / \mathrm{h}): 120,110,100,90$, $80,70,60,50,40,30,20,10$; and critical speeds for the speeds adjustment process $(\mathrm{km} / \mathrm{h}): 65,45,35,30$. 


\begin{tabular}{|l|c|c|}
\hline & Midnight & $\mathbf{8}$ am \\
\hline Running time for TIBDP & $12 \mathrm{~h} 33 \mathrm{~m} 47 \mathrm{~s}$ & $12 \mathrm{~h} 34 \mathrm{~m} 31 \mathrm{~s}$ \\
\hline Running time for NHA & $1 \mathrm{~m} 17 \mathrm{~s}$ & $5 \mathrm{~m} 25 \mathrm{~s}$ \\
\hline No. of Same Routes & 201 & 145 \\
\hline No. of Routes NHA Better Than TIBDP & 210 & 175 \\
\hline Mean emissions for TIBDP & $4459.8 \mathrm{~g}$ & $4666.4 \mathrm{~g}$ \\
\hline Mean emissions for NHA & $4380.4 \mathrm{~g}$ & $4623.0 \mathrm{~g}$ \\
\hline
\end{tabular}

Table 3 Results obtained by NHA and TIBDP

As shown in Table 3, the running time presented in each cell is the total computational time for generating the 210 routes with a specified starting time. The running time for the time-increment based DP is more than 12 hours. However, for the new heuristic method, it is only several minutes.

The quality of solution from the new heuristic method is better than the TIBDP, even though it takes much less computing time. Among the 210 routes, 201 routes obtained by these two methods are the same when the starting time is midnight, and all of the solutions proposed by the new heuristic method are better than that of the time-increment based DP, which is on the average $1.81 \%$ less in terms of the objective value. In the case of starting at $8 \mathrm{am}$, the fuel emissions obtained by the NHA are $0.94 \%$ less than those obtained by the TIBDP on average. However, 35 solutions from the new heuristic method are inferior to that from time-increment based DP with the worst case being 3.69\%.

Although there is a difference in the assumptions dealing with speeds for arcs crossing time slots for the two models, each route in the case study will only cross up to about 3 time slots, thus differences caused by such assumptions will be small. From the theoretical point of view, the TIBDP should get solutions closer to the optimal solution than the NHA. Especially when the time increment is quite small, TIBDP can be considered an exact method. However, there are $37.35 \%$ arcs shorter than $50 \mathrm{~m}$ in practice, and the fuel emissions for those short arcs may be overestimated by TIBDP. This is the main reason why the new heuristic approach provides solutions with 
higher quality than the time-increment based DP in this test.

\subsubsection{Least Polluting Routes compared with Fastest Routes}

The least fuel emission routes obtained by NHA are compared with the shortest-time route obtained by the Road Timetable ${ }^{\mathrm{TM}}$ in terms of fuel emissions, time and distance. Midnight and 8am again are used to represent the different starting times. The values in the table show the ratio of the quantity measured in each row when minimizing fuel emissions compared to that quantity when minimizing travel time.

\begin{tabular}{|l|c|c|c|}
\hline & & Midnight & $\mathbf{8 ~ a m}$ \\
\hline \multirow{2}{*}{ No. of Same Routes } & & 102 & 93 \\
\hline \multirow{2}{*}{ Fuel Emission } & Average & 0.9354 & 0.9463 \\
\cline { 2 - 4 } & Extreme Case & 0.6707 & 0.6655 \\
\hline \multirow{2}{*}{ Travel Time } & Average & 1.1033 & 1.0854 \\
\cline { 2 - 4 } & Extreme Case & 1.3089 & 1.4842 \\
\hline \multirow{2}{*}{ Distance } & Average & 0.9534 & 0.9315 \\
\cline { 2 - 4 } & Extreme Case & 0.6915 & 0.6173 \\
\hline
\end{tabular}

Table 4 Comparison of least-polluting routes to shortest time routes

As shown in Table 4, even with different objectives, nearly half of the routes are exactly the same. The differences with regard to the total fuel emissions, travel time and distance for time optimization solution and emission optimization solution are presented. In addition to the average difference, extreme cases are examined as well.

On average, about 6-7\% savings in fuel emissions can be achieved by the least polluting model, on top of the $7 \%$ of $\mathrm{CO} 2$ saving made by taking the time-varying speeds into account in the shortest time model [11]. However, in terms of the average travel time, the least polluting model requires about 9-10\% more trip time than the shortest-time model. Besides, the trip distance proposed by the least polluting model 
is also about 5-6\% shorter than that of the shortest-time model. In some extreme cases, the fuel emissions can be reduced up to $33 \%$. But the time to complete the trip may increase by $48 \%$ on some occasions.

\section{Conclusions}

In this paper, a least polluting problem for a single route in a network with time-varying speeds is addressed, which consider speeds as well as routes as decision variables. Two methods are developed to solve the problem, and both of them involve DP recurrences. One is called time-increment based dynamic programming, and the other is a new heuristic approach. Compared with the time-increment based DP, the new heuristic approach is competitive in both solution quality and running time. A primary cause of poor quality solution of the time-increment based DP is that the traffic data used in the experiment is made up of relatively short arcs. The results indicated that a DP algorithm, which has also been studied by other researchers, might not be an efficient method to solve the fuel emission optimization problem in networks with short arcs. As the single route solving technique has to be embedded into a heuristic method framework to solve the full VRP, the new heuristic approach is more appropriate.

Provided time-dependent speeds, customers' sequence and other network information are known in advance, such a single route solving technique is able to suggest a least polluting vehicle scheduling plan, including which routes to choose, what are the proper speeds to travel and how long to wait at customer nodes if necessary. A test with the Bristol Data set has indicated further savings in fuel emissions can be achieved in the model, on top of the savings made by using variable travel time information in the time optimization model [11].

As part of the VRP solving algorithm, the new heuristic approach can be utilized in two ways. One is to be embedded into a heuristic method framework, such as a column generation based tabu search algorithm, to solve the full VRP. For each new sequence of customers (column) generated, the corresponding cost can be calculated by the new heuristic method. The second is for the new heuristic method to be used as a post-process to calculate the optimal fuel emissions for a pre-determined sequence of customers in a complete route. In a real-world scheduling environment, this single 
route solving technique can be applied to improve the fuel emissions based on the current customer sequences.

\section{Acknowledgements}

The first author was supported by a grant under the LANCS Initiative from the EPSRC. (Grant number: EP/F033613/1). 


\section{REFERENCES}

[1] A.K. Ziliaskopoulos, H.S. Mahmassani, A time-dependent shortest path algorithm for real-time intelligent vehicle highway systems, Transport Res Rec 1408 (1993), 94-104.

[2] I. Chabini, Discrete dynamic shortest path problems in transportation applications: complexity and algorithms with optimal run time, Transport Res Rec 1645 (1998), $170-175$.

[3] I.S. Dolinskaya, Optimal path finding in direction, location and time dependent environments, PhD thesis, The University of Michigan, 2009.

[4] B-H. Ahn and J-Y. Shin, Vehicle-routeing with time windows and time varying congestion, J Oper Res Soc, 42 (1991), 393-400.

[5] M.E.T. Horn, Efficient modeling of travel in networks with time-varying link speeds, Networks 36 (2000), 80-90.

[6] K. Sung, M. Bell, M. Seong, and S. Park, Shortest paths in a network with time-dependent flow speeds, Eur J Oper Res 121(2000), 32-39.

[7] S. Ichoua, M. Gendreau, J-Y. Potvin, Vehicle dispatching with time-dependent travel times, Eur J Oper Res 144 (2003), 379-396.

[8] R.W. Eglese, W. Maden, A. Slater, A road timetable to aid vehicle routing and scheduling, Comput Oper Res 33 (2006), 3508-3519.

[9] T. Van Woensel, L. Kerbache, H. Peremans, N. Vandaele, Vehicle routing with dynamic travel times: A queueing approach, Eur J Oper Res 186 (2008), 990-1007.

[10] B. Fleischmann, M. Gietz, S. Gnutzmann, Time-varying travel times in vehicle routing, Transport Sci 38 (2004), 160-73.

[11] W. Maden, R.W. Eglese, D. Black, Vehicle routing and scheduling with time-varying data: A case study, J Oper Res Soc 6 1(2010), 515-522.

[12] A.L. Kok, E.W. Hans, J.M.J. Schutten, Optimizing departure times in vehicle routes, Eur J Oper Res, 210 (2011), 579-587.

[13] S. Kim, M.E. Lewis, C.C. III White, Optimal vehicle routing with real-time traffic information, Intell Transp Sy 6 (2005), 178-188. 
[14] S. Kim, M.E. Lewis, C.C. III White, State space reduction for nonstationary stochastic shortest path problems with real-time traffic information, Intell Transp Sy 6 (2005), 273-284.

[15] A. Haghani and S. Jung, A dynamic vehicle routing problem with time-dependent travel times, Comput Oper Res 32 (2005), 2959-2986.

[16] European Environment Agency (EEA), Transport and environment: on the way to a new common transport policy, EEA Report No. 1/2007, Copenhagen, 2007.

[17] Committee on Climate Change (CCC), Committee on climate change-Annual report \& accounts-1 April 2010 to 31 March 2011, [online] London: The Stationery Office., 2011, Available at:

<http://downloads.theccc.org.uk.s3.amazonaws.com/Annual\%20Report\%20\&\%20Ac counts\%202011/1427_CCC-AnnualReport-2010_6_bookmarks.pdf > [Accessed 02 October 2011].

[18] R. Dekker, J. Blowmhof, I. Mallidis, Operations research for green logistics- An overview of aspects, issues, contributions, and challenges, Eur J Oper Res 219 (2012), 671-679.

[19] C. Paterson, Carbon Footprint Methodology \& Model, Master dissertation, Lancaster University, 2007.

[20] D.A. Hensher, Climate change, enhanced greenhouse gas emissions and passenger transport - What can we do to make a difference? Transport Res D-Tr E 13 (2008), 95-111.

[21] T. Bektas and G. Laporte, The pollution-routing problem, Transport Res B-Meth 45 (2011), 1232-1250.

[22] S. Ubeda, F.J. Arcelus, J. Faulin, Green logistics at Eroski: A case study, Int J Prod Econ, 131 (2011), 44-51.

[23] S. Erdoğan, E. Miller-Hooks, A green vehicle routing problem, Transport Res E-Log, 48 (2012), 100-114.

[24] M.A. Figliozzi, Vehicle routing problem for emissions minimization, Transportation Res Rec 2197 (2010), 1-7.

[25] M.A. Figliozzi, The impacts of congestion on time-definitive urban freight distribution networks CO2 emission levels, Transport Res C-Emer, 19 (2011), 766-778. 
[26] Department for Transport, Road vehicle emission factors 2009, [online], 2009, Available at: 〈http://www2.dft.gov.uk/pgr/roads/environment/emissions/regulated.xls $>$ [Accessed 03 September 2011]

[27] Department for Environment Food and Rural Affairs, Guidelines to Defra / DECC's greenhouse gas conversion factors for company reporting, [online], 2009, Available at:

$<$ http://archive.defra.gov.uk/environment/business/reporting/conversion-factors.htm $>$ [ Accessed 16 Jun 2011]

[28] H. Hashimoto, T. Ibaraki, S. Imahori, M. Yagiura, The vehicle routing problem with flexible time windows and traveling times, Discrete Appl Math 154 (2006), 2271-2290. 